\title{
INNER PRODUCTS CHARACTERIZED BY DIFFERENCE EQUATIONS
}

\author{
GORDON G. JOHNSON
}

ABSTRACT. A normed linear space $X$ is an inner product space iff, for some integer $k \geqq 3, \sum_{t=0}^{k}\left(\begin{array}{c}k \\ t\end{array}\right)(-1)^{t}\|a+a b\|^{2}=0$ for all $a$ and $b$ in $X$.

THEOREM. If $X$ is a linear space with norm $\|\cdot\|$ and, for some integer $k \geqq 3$,

$$
\sum_{t=0}^{k}\left(\begin{array}{l}
k \\
t
\end{array}\right)(-1)^{t}\|a+t b\|^{2}=0
$$

for all $a, b \in X$ then $\sum_{t=0}^{k}\left(\begin{array}{c}k \\ t\end{array}\right)(-1)^{t}\|a+t b\|^{2}=0$ for every integer $k \geqq 3$ and $\|\cdot\|$ is induced by an inner product on $X$.

Definition. Suppose $X$ is a normed linear space and $k$ and $n$ are nonnegative integers. Let

$$
D_{k}^{n}(a, b)=\sum_{t=0}^{k}\left(\begin{array}{l}
k \\
t
\end{array}\right)(-1)^{t}\|a+(t+n) b\|^{2}
$$

where $a$ and $b$ are in $X$ and $\|\cdot\|$ denotes the norm on $X$.

PROOF. Suppose $k$ is an integer greater than 2 and $D_{k}^{0}(a, b)=0$ for all $a$ and $b$. Then $D_{k}^{n}(a, b)=0$ if $n$ is a nonnegative integer; moreover, $D_{k}^{n}(a, b)=$ $D_{k-1}^{n+1}(a, b)-D_{k-1}^{n}(a, b)$ and hence $D_{k-1}^{n}(a, b)=D_{k-1}^{0}(a, b)$.

Suppose $m$ is a positive integer not exceeding $k$ then by iteration we have that

$$
D_{k-m}^{n}(a, b)=\sum_{t=0}^{m-1}\left(\begin{array}{l}
n \\
t
\end{array}\right) D_{k-m+t}^{0}(a, b) \text { for } n=0,1,2, \cdots,
$$

and hence

$$
D_{0}^{n}(a, b)=\sum_{t=0}^{k-1}\left(\begin{array}{l}
n \\
t
\end{array}\right) D_{t}^{0}(a, b) \quad \text { for } n=0,1,2, \cdots .
$$

Recall that $D_{0}^{n}(a, b)=\|a+n b\|^{2}$. Hence it follows that

$$
\|(1 / n) a+b\|^{2}=\sum_{t=0}^{k-1}\left(\begin{array}{l}
n \\
t
\end{array}\right) D_{t}^{0}(a, b) / n^{2} .
$$

Received by the editors April 20, 1972 and, in revised form, June 21, 1972. AMS (MOS) subject classifications (1970). Primary 46C10; Secondary 39A25. 
We have then that

$$
\begin{aligned}
\lim _{n \rightarrow \infty} & \|(1 / n) a+b\|^{2}=\|b\|^{2} \\
= & \lim _{n \rightarrow \infty} \frac{1}{n^{2}} \sum_{t=0}^{k-1}\left(\begin{array}{l}
n \\
t
\end{array}\right) D_{t}^{0}(a, b) \\
= & \lim _{n \rightarrow \infty}\left[D_{0}^{0}(a, b) / n^{2}+\left(\left(\begin{array}{l}
n \\
1
\end{array}\right) / n^{2}\right) D_{1}^{0}(a, b)+\left(\left(\begin{array}{l}
n \\
2
\end{array}\right) / n^{2}\right) D_{2}^{0}(a, b)\right. \\
& \left.\quad+\left(\left(\begin{array}{l}
n \\
3
\end{array}\right) / n^{2}\right) D_{3}^{0}(a, b)+\cdots+\left(\left(\begin{array}{c}
n \\
k-1
\end{array}\right) / n^{2}\right) D_{k-1}^{0}(a, b)\right] .
\end{aligned}
$$

In order that this limit exist it is necessary that $D_{l}^{0}(a, b)=0$ if $3 \leqq l \leqq k-1$. Hence the limit is $\frac{1}{2} D_{2}^{0}(a, b)$ if $k \geqq 3$. Therefore

$$
\|b\|^{2}=\frac{1}{2}\left[\|a+2 b\|^{2}-2\|a+a b\|^{2}+\|a\|^{2}\right]
$$

for all $a$ and $b$ in $X$, which is a simple reformulation of the parallelogram law.

Hence the space is an inner product space from which it is easy to establish that $D_{k}^{0}(a, b)=0$ if $k$ is any integer greater than 2 .

\section{REFERENCE}

M. M. Day, Normed linear spaces, Ergebnisse der Mathematik und ihrer Grenzgebiete, N.F., Heft 21, Academic Press, New York; Springer-Verlag, Berlin, 1962. MR 26 \#2847.

Department of Mathematics, University of Houston, Houston, TeXas 77004 